\title{
Erratum to: Bursty Human Dynamics
}

\section{Erratum to: \\ M. Karsai et al., Bursty Human Dynamics, SpringerBriefs in Complexity, https://doi.org/10.1007/978-3-319-68540-3}

The original version of the book was inadvertently published without the following corrections:

In Chap. 4, Pg. 60, 6th line from last, the space between " $\lambda_{n} t_{p}$ " and "," has been removed.

In reference [257], the incorrect arXiv number "arXiv:1307.0814" has been corrected as "arXiv:1209.1411".

In reference [267], the incorrect arXiv number "arXiv:0510216" has been corrected as "arXiv:physics/0510216".

In reference [125], the title "Community-based static modeling, stylized facts in social networks" has been changed as "Stylized facts in social networks: Community-based static modeling".

The updated online version for this book can be found at https://doi.org/10.1007/978-3-319-68540-3_4

https://doi.org/10.1007/978-3-319-68540-3 